\title{
Digital radiograph rejection analysis during "Coronavirus disease 2019 (COVID-19) pandemic" in a tertiary care public sector hospital in Khyber Pakhtunkhwa Province of Pakistan
}

\author{
Amir Ali $^{1} \cdot$ Muhammad Yaseen $^{1}$ (D)
}

Received: 25 January 2021 / Revised: 20 April 2021 / Accepted: 20 May 2021 / Published online: 7 June 2021

(c) The Author(s), under exclusive licence to Springer Nature Singapore Pte Ltd. 2021

\begin{abstract}
Background Evaluation of X-ray reject analysis is an important quality parameter in diagnostic facility. The aim of this study was to find out the radiograph rejection and its causes during the coronavirus disease 2019 (COVID-19) pandemics as there was fear of coronavirus disease infection among the technical staff from the incoming patients in a busy, high volume public sector tertiary care hospital.

Materials and method This descriptive study was conducted at Radiology Department, Lady Reading Hospital, Peshawar from August to November, 2020. The rejected radiographs and their causes were analyzed.

Results A total of 15,000 X-ray procedures were conducted during study period out of which 2550 cases were repeated making the total rejection $17 \%$. Rejection in male and female were 74.3 and $25.7 \%$, respectively, while rejection in adults was $(80.1 \%)$ and $(19.9 \%)$ in pediatric age group of the total rejection. The main cause of rejection was positioning $(30.5 \%)$ followed by artifacts (22.4\%), motion (12.1\%), improper collimation (10\%), wrong labeling (8.4\%), exposure errors (6.9\%), detector errors (3.7\%), machine faults (2.8\%), re-request from referring physician (1.7\%), and PACS issues (1.5\%). In terms of body anatomical parts, the highest rejection was observed in extremities (44.1\%), followed by chest radiography (23.3\%), spine (11.4\%), abdomen (6.4\%), skull (5.9\%), pelvis (4.7\%), KUB (3.7\%), and neck (0.6\%), respectively.

Conclusion Radiograph rejection is common problem in every diagnostic facility but significant reduction can be achieved by implementing rejection analysis as basic quality indicator, and conducting technologist/s specific training programs for their knowledge and skill enhancement.
\end{abstract}

Keywords Quality assurance $\cdot$ Quality control $\cdot$ Picture archiving and communication system $\cdot$ Artifacts $\cdot$ Rejection analysis program

\section{Introduction}

Ionizing radiations are employed in diagnostic imaging to perform various procedures of patients to produce good quality radiographs which help in diagnosis and treatments of various diseases. The use of radiation is beneficial to patient if it is clinically justified (if the benefits of procedure overweight's the risk associated with the use of radiations).

Muhammad Yaseen

myaseena@hotmail.com

1 Department of Radiology, Lady Reading Hospital (Medical Teaching Institute) Peshawar, Soekarno Rd, PTCL Colony, Peshawar 25000, Pakistan
In diagnostic imaging, one of the main goals of a quality assurance (QA) program is to produce consistent highquality radiographs at a minimum exposure to the patient [1]. The employment of reject analysis as part of overall Quality Assurance (QA) programs in clinical radiography and radiology services in the evaluation of image quality is a well-established practice [2]. The use of radiations to obtain high quality radiography is a complex process and needs adequate skills and dedicated training of the performing technologists as both the patient and staff are exposed to unwanted radiation exposure if proper optimization protocols and basic radiation protection principles are not employed in daily routine practices. The detrimental effect of ionizing radiations can be reduced by properly adhering radiation protection principles in accordance with ALARA (As low as reasonably achievable) principle. Better staff 
protection is associated with better patient protection and the repetition of any procedure pose a certain threat of high radiation doses to patient as well as staff.

Patients might undergo repeated X-ray examinations after their initial X-ray radiographs are rejected due to different reasons such as poor image quality, artifact, anatomical cutoff, patient motion, and equipment malfunction [3]. Rejected radiographs that are repeated, may lead to extra radiation dose to the patient; reduction in the lifetime of the X-ray machines, which added expenses on the health care system; and long waiting list $[4,5]$.

The rejection rate in screen film radiography and then computed radiography were believed to be higher in number perhaps due to too much manual interventions and also the narrow exposure latitude of film/screen results in the high rejection rates for under/over exposed films. On the other hand, digital systems were assumed to have reduced the rejection due to more forgiving exposure latitude [6]. In addition to the wider exposure latitude, the advantages of digital over analog systems include processing capabilities and manipulation, which allow technologists to adjust radiographs quality [7]. Reject radiographs are still a problem with digital radiography system as it is easy in terms of acquisition and facilitate the repetition of images specially for flat panel technologies.

In digital systems, radiographs are more likely to be rejected due to positioning errors or artifacts [8]. Therefore, reject analysis program (RAP) is a basic quality indicator used in diagnostic radiology services to evaluate the quantity of reject radiographs and find the causes behind. This will assist us to find ways and improve the identified weak zones to further reduce the rejection rate ensuring minimum doses to patients, lowering extra cost involved and to prolong the life of X-ray machines.

Determination of rejection rate is important for any facility for optimum image quality with reduced patient and staff doses but we felt the need to evaluate it especially during the coronavirus disease 2019 (COVID-19) pandemics as there was fear of infection where every person seems to be a suspect as certain patients do not show any signs and symptoms which even becomes difficult to distinguish as compared to those having symptoms as that of coronavirus disease 2019 (COVID-19). The technical staff were reluctant to come close to patients, touch and position them as per set protocols despite having adequate safety precautions against coronavirus disease 2019 infection, i.e., masks, gloves, gown etc. Thus the technical staff were trying to position the patients from a distance through verbal instructions without properly positioning them as per set protocols which tend to result in high rejection as observed as patients have no idea of selfpositioning and orientation.

Thus the basic purpose of this study was to evaluate the rejection rate of radiographs in general as well as the contribution of coronavirus disease infection factor in particular along with finding out the causes behind rejection so, that we can identify those weak areas where improvement can be made towards good quality images with minimum patient doses along with skill enhancement of our staff.

\section{Materials and methods}

Lady Reading Hospital is the largest public sector hospital in the city of Peshawar catering services to huge volume of patient coming from all over the province. This descriptive study was performed in radiology department during the period from August to November, 2020 during which a total of 15,000 plain radiographs were performed on four (4) dedicated digital X-ray radiographic systems installed in the main radiology department of the hospital.

1. Two (2) Hitachi X-ray machines (Model Radnext-50), $150 \mathrm{kV}$ and $500 \mathrm{~mA}$ each

2. One (1) Philips Healthcare X-ray system (Model Dura Diagnostics), $150 \mathrm{kV}$ and $1000 \mathrm{~mA}$

3. One (1) Matora X-ray system (Model Virsaris Vision-V), $150 \mathrm{kV}$ and $500 \mathrm{~mA}$

All radiographs were performed on referring physician online feed request received at main counter beside having written request as radiation exposure was clinically justified with necessary protection measures were also given to patients.

The rejection rate was calculated using the formula;

Rejection rate $(\%)=\frac{\text { Number of rejected films }}{\text { Total number of procedures (films) }} \times 100$.

\section{Statistical analysis}

To check the inter observer variability and agreement between the radiographer and radiologist kappa statistics was applied on a total of 160 images subdivided into 20 images per body part (extremities, chest radiograph, spine, KUB, abdomen, pelvis, skull, and neck), respectively, as per our study and the result showed substantial agreement with a score in between (0.61-0.80). Based on strong agreement between radiologist and radiographer, the rejection was mainly done by the radiographer.

No complex statistical analysis was required for this study. The data were entered into Microsoft excel and results were expressed in percentages and graphs. 


\section{Criteria of rejection}

The rejection was mainly done by the qualified radiographer who has experience of more than 15 years in medical imaging along with the reporting radiologist who is at assistant professor level having experience of more than 10 years and the images of every conducted procedure were viewed and analyzed through PACS. However, to check the inter observer variability and agreement between the radiographer and radiologist kappa statistics was applied on a total of 160 images subdivided into 20 images per body part (Extremities, Chest radiograph, Spine, KUB, Abdomen, Pelvis, Skull and Neck), respectively, as per our study and the result showed substantial agreement with a score in between (0.61-0.80).

As the radiographer is responsible for performing the procedures, so the decision of radiograph rejection was made by that radiographer based on his professional judgment. In few instances, where the radiographer was not sure, then the decision of rejection was made by the reporting radiologist. The number and causes of rejection were compiled in a specially designed data collection performa in accordance with the ten (10) predefined and standardized causes of rejection as per the report of AAPM Imaging Physics Committee Task Group 151; positioning, artifacts, patient motion, collimation, labeling, exposure levels, detector errors, machine faults, re-request from referring physician and PACS issues.

\section{Results}

Out of the total 15,000 diagnostic procedures performed during the study period of August-November, 2020, during which the Coronavirus disease 2019 (COVID-19) pandemic was under control in Pakistan, the gender distribution shows 10,125 (67.5\%) males and $4875(32.5 \%)$ female patients while as per age group classification $11,200(74.7 \%)$ were adults and $3800(25.3 \%)$ patients were pediatric (age $<10$ years as per hospital policy) while the distribution of procedures performed with respect to body parts were; Extremities 7548 (50.3\%), i.e., both upper and lower extremities were taken collectively as both have many separate sub parts. Chest 3858 (25.7\%), spine $1661(11.1 \%)$, KUB 609 (4.1\%), abdomen $575(3.8 \%)$, pelvis $342(2.3 \%)$, skull $336(2.2 \%)$, and neck $71(0.5 \%)$ as shown in Fig. 1.

To check the inter observer variability and agreement between the radiographer and radiologist regarding the rejection of certain procedures, kappa statistics was applied on a total of 160 images subdivided into 20 images per body part (extremities, chest radiograph, spine, KUB, abdomen, pelvis, skull, and neck), respectively, as per our study and the result showed substantial agreement with a score in between $(0.61-0.80)$.

The rejected radiographs during the study period were 2550. Out of which the rejected radiographs for male were $1895(74.3 \%)$ while $655(25.7 \%)$ rejected radiographs in females. The rejection was $(80.1 \%)$ in adults and $(19.9 \%)$ in pediatric age patients as per age group classification. The most common cause of rejection in our study was positioning $30.5 \%$ followed by artifacts $22.4 \%$, motion $12.1 \%$, improper collimation $10 \%$, wrong labeling $8.4 \%$, exposure errors $6.9 \%$, detector errors $3.7 \%$, machines faults $2.8 \%$, re-request from referring physician $1.7 \%$ and PACS issues 1.5\%, respectively, from Fig. 2.

In-terms of body parts, the highest reject radiographs were observed in extremities $44.1 \%$ followed by chest radiography $23.3 \%$, spine $11.4 \%$, abdomen $6.4 \%$, skull $5.9 \%$, pelvis $4.7 \%$, KUB $3.7 \%$, and neck $0.6 \%$, respectively, from Fig. 3.

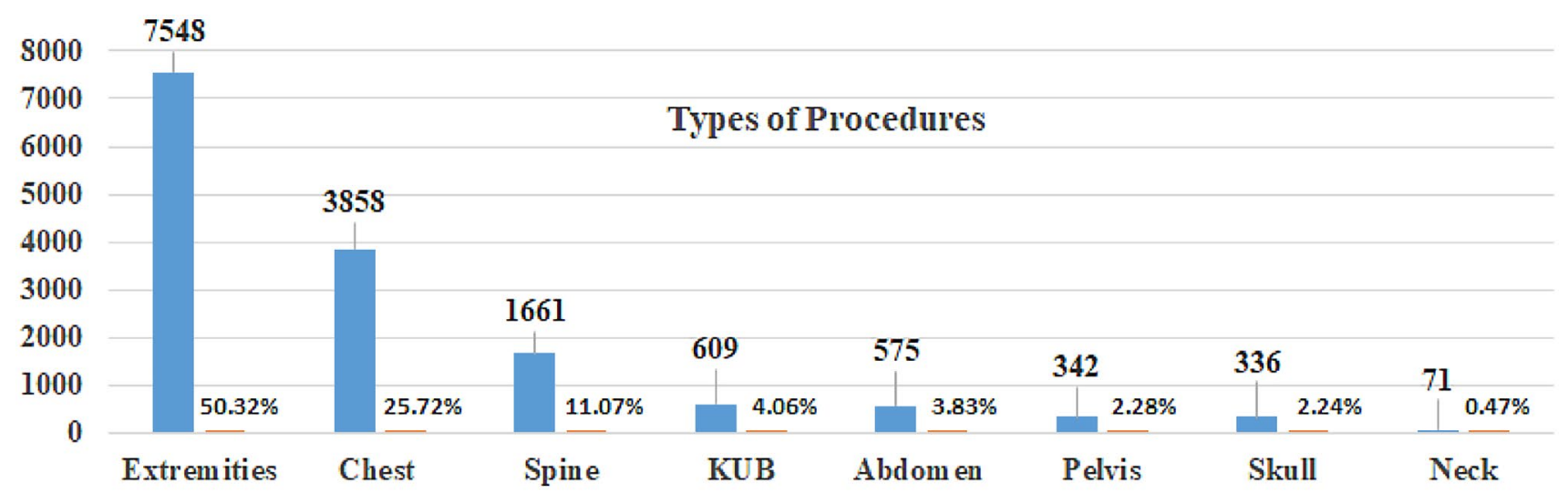

Fig. 1 Distribution of procedures performed as per anatomical body parts from August to November, 2020 


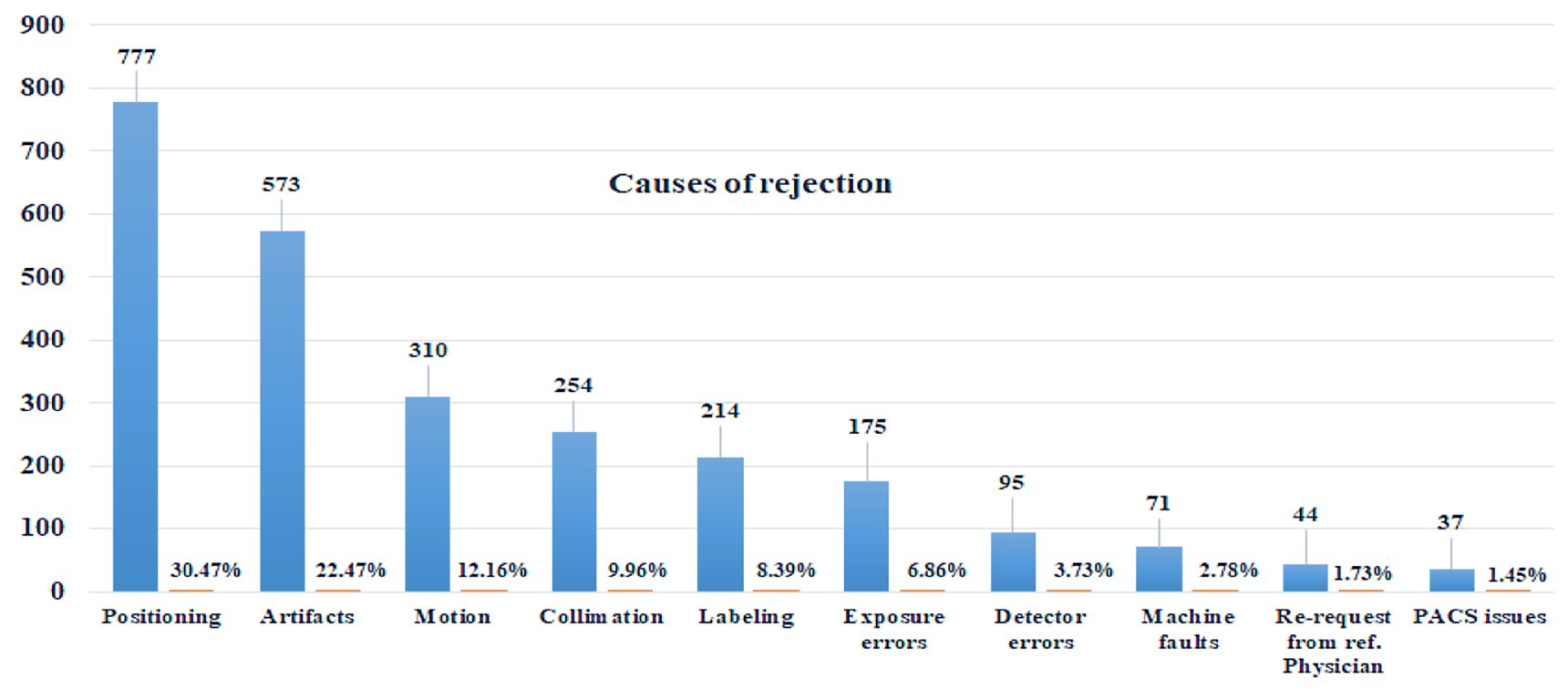

Fig. 2 Rejected radiographs with respect to causes of rejection from August to November, 2020

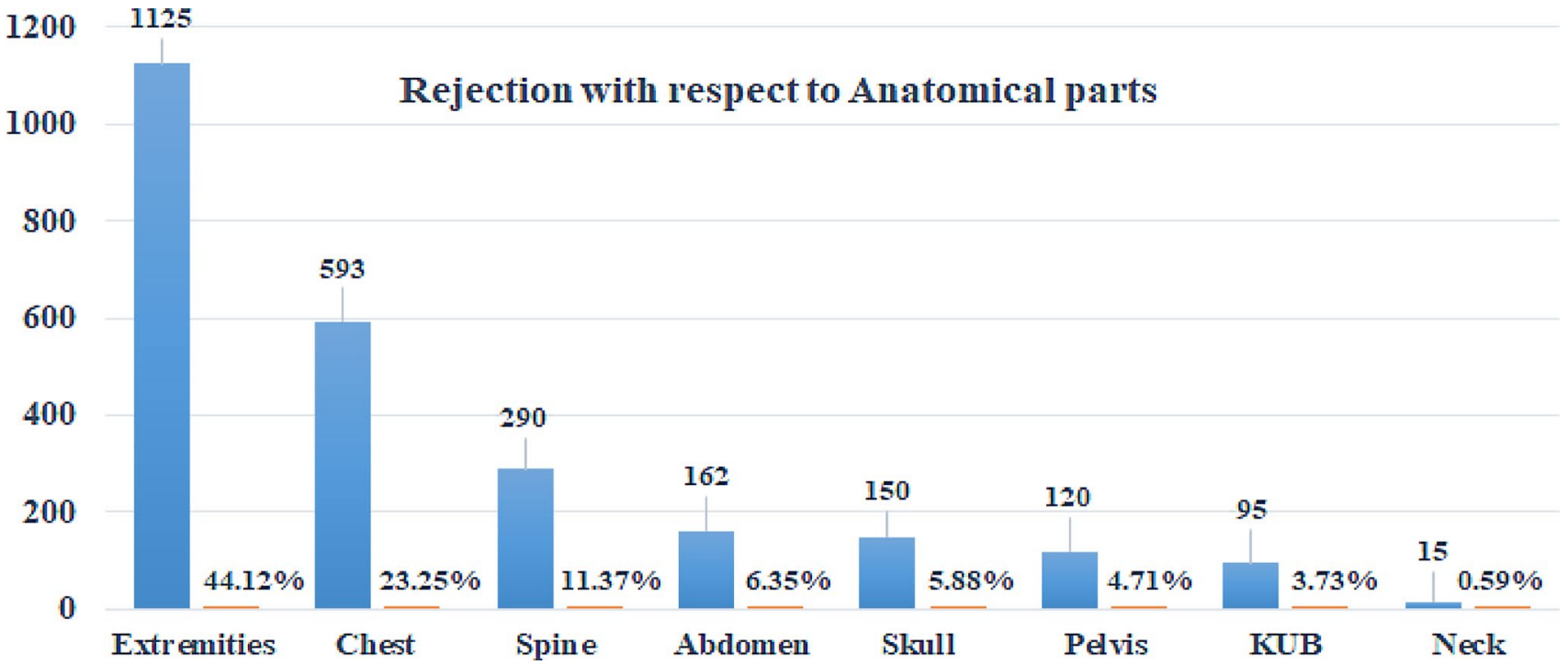

Fig. 3 Rejected radiographs as per anatomical body parts from August to November, 2020

\section{Discussion}

Reject analysis is an important part of quality assurance programs in a radiology department providing radiography services to ensure reduction in the factors responsible for rejects and thus to reduce the cost, workload, and radiation exposure to patients and personnel [9]. The analysis of rejected radiographs provides useful information about the rejection rate of the facility and more importantly, the underlying causes for rejection which can be addressed in a better way to improve the image quality, lower the rejection rate, decrease patient radiation exposure, cut down cost, optimize machine performance, and life along with reduction of staff burden.

The rejection in our study was $17 \%$ which is higher than other relevant studies reporting $11-12 \%$ in Norway $[4,10]$ and $11.15 \%$ in Iran [11]. The rejection in our study was high because of fear from coronavirus disease infection, improper implementation of patient preparation protocols, poor reflection of technical staff skills and techniques and other causes of rejection as shown in (Fig. 2).

One important aspect of our study was to find out the impact of coronavirus disease 2019 (COVID-19) pandemics 
on the rejection rate and attitude of technical staff who were performing the procedures of patients. It was found that the fear of coronavirus disease infection among technical staff contributed largely to positioning errors, anatomical cutoffs and unwanted patient movements specifically as technicians having age above forty (40) years were reluctant to come into close proximity during positioning of patients despite having all the safety measures against coronavirus disease infection and were trying to position them verbally instructing to orient themselves accordingly. This resulted in large number of rejection of radiographs as patient has no idea of self-positioning and orientation. coronavirus disease 2019 (COVID-19) pandemics contributed largely in rejection of radiographs but the exact percentage increase cannot be estimated due to lack of similar studies conducted before the pandemics, however, we will conduct similar study once the pandemics is over to calculate the exact rejection in normal working conditions.

The main cause of rejection was positioning $44.1 \%$ followed by artifacts $22.5 \%$. Artifacts were the second largest cause of rejection arising from the pockets items of male patient and the extended use of jewelry as part of tradition especially among females, i.e., hair pins, hair clips, and accessories of females contributing towards rejection which reflects poor implementation of preparation protocols, low literacy rate and low socio-economic conditions of incoming patient and the large influx of patients which resulted in anxiety among the technical staff while rare use of immobilization devices and support to unstable patients mainly in traumatic, un-cooperative patients, adults of high age and children of low age who were unable to hold their position resulted in rejection as well. Exposure errors were remarkably less as the use of digital systems provides extended dynamic range for exposure minimizing exposure errors and are consistent with other reported data with respect to causes of rejection (Table 1).

Among the various types of examination performed, extremities reported the highest number of rejection (44.1\%) followed by chest radiography reporting (23.3\%) while spine showing $11.4 \%$ rejection along with other body parts (Fig. 3). High rejection in extremities was reported as it was the highest performed examination $(50.3 \%)$ along with other contributing factors like positioning errors specially due to coronavirus disease 2019 (COVID-19) pandemic fear, patient movement, labeling errors and anatomical cut off. Chest radiography rejections were mainly due to artifacts and positioning errors both in male and females, breath holding problems specially in high age adults and motion in pediatric patients while spine rejections resulting from artifacts specially in male due to pocket items, exposure issues and motion, abdomen (5.9\%) again appearance of artifacts, techniques problems and image contrast issues, skull (5.9\%) possibly due to positioning difficulties and proper projection angles settings, pelvis (4.7\%) and KUB (3.7\%) rejected due to poor implementation of patient preparation protocols, artifacts, and improper collimation while neck $(0.6 \%)$, due to movement and not using immobilization and are consistent with relevant published data (Table 2).

From the comparison of rejected radiographs between adults and pediatric age groups, it was found that contribution of rejection in adults was significant in total rejection as compared to Peads (Fig. 4). However, the rejection due to motion $(5.3 \%)$ and positioning (4.4\%) were relatively high as compared to other less significant causes specially
Table 1 Comparison of causes of rejected radiographs with relevant studies

Table 2 Comparison of rejected radiographs in terms of anatomical body parts with relevant studies

\begin{tabular}{|c|c|c|c|c|c|c|}
\hline \multirow[t]{2}{*}{ Causes of rejection } & \multicolumn{2}{|c|}{ Current study } & \multicolumn{2}{|c|}{ Alyousef et al. [3] } & \multicolumn{2}{|c|}{ Chih-Sheng et al. [12] } \\
\hline & $n$ & $\%(N=2550)$ & $n$ & $\%(N=455)$ & $n$ & $\%(N=4812)$ \\
\hline Positioning & 777 & 30.5 & 64 & 14 & 2697 & 56.1 \\
\hline Artifact & 573 & 22.4 & 59 & 13 & 990 & 20.6 \\
\hline Exposure error & 175 & 6.9 & 27 & 6 & 193 & 4 \\
\hline
\end{tabular}

\begin{tabular}{|c|c|c|c|c|c|c|}
\hline \multirow[t]{2}{*}{ Body parts } & \multicolumn{2}{|c|}{ Current study } & \multicolumn{2}{|c|}{ Alyousef et al. [3] } & \multicolumn{2}{|c|}{ Weatherburn et al. [13] } \\
\hline & $n$ & $\%(N=2550)$ & $n$ & $\%(N=455)$ & $n$ & $\%(N=525)$ \\
\hline Extremities & 1125 & 44.1 & 196 & 43 & 105 & 20 \\
\hline Chest & 593 & 23.3 & 141 & 31 & 235 & 44.8 \\
\hline Spine & 290 & 11.4 & 36 & 8 & 88 & 16.8 \\
\hline Abdomen & 162 & 6.4 & 41 & 9 & 40 & 7.6 \\
\hline Skull & 150 & 5.9 & 18 & 4 & 47 & 8.9 \\
\hline Pelvis & 120 & 4.7 & 23 & 5 & 10 & 1.9 \\
\hline KUB & 95 & 3.7 & - & - & - & - \\
\hline Neck & 15 & 0.6 & - & - & - & - \\
\hline
\end{tabular}




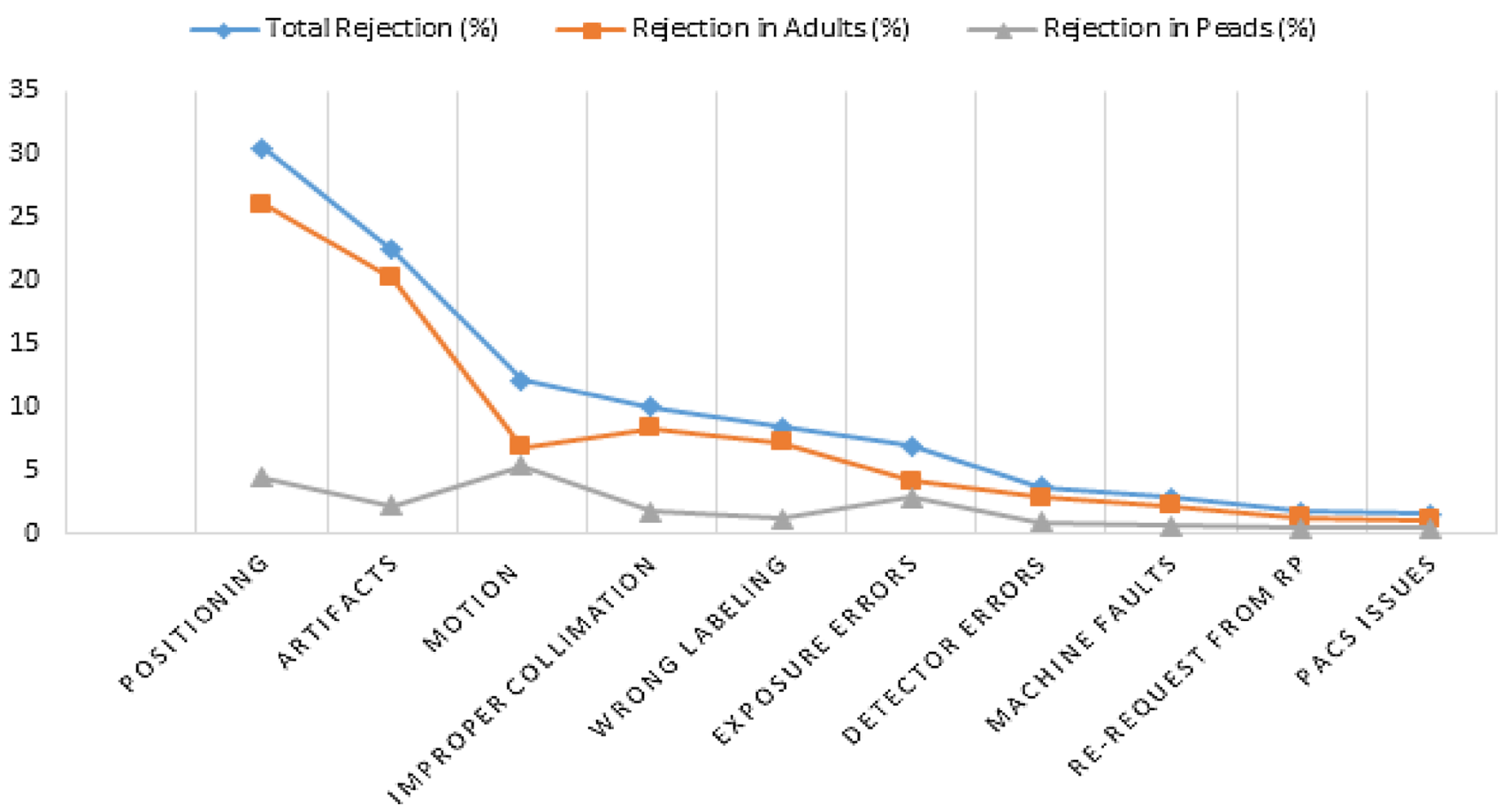

Fig. 4 Comparison of rejection in adults and Peads with respect to causes of rejection

in pediatrics because of the factor that these age groups are relatively non-cooperative, difficult to position due to frequent motion during procedures as they cannot sustain their position without proper support. Positioning (26.1\%), artifacts (20.2\%), improper collimation (8.3\%), and motion $(6.8 \%)$ were the significant causes of rejection in adults specifically due to fear factors, improper implementation of patient preparation protocols, discomfort among staff due to huge influx of patients etc.

While on comparison of rejected radiographs in terms of body parts, the rejection in adult's body parts were far higher than pediatrics for the reason that the number of adults cases were higher (74.7\%) as compared to $(25.3 \%)$ of pediatrics age group of total cases conducted during the study period and second, due to the positioning errors, motion, collimation errors, fear factors, discomfort, and artifacts which were more prominent in adults as compared to pediatrics age groups (Fig. 5).

As image retake analysis is a basic quality control (QC) indicator and is used for quality assurance in digital radiography. Therefore, the AAPM recommend rejection analysis to be performed on at least on quarterly basis, but preferably monthly [1]. The results obtained from this study will be analyzed critically as per the staff requirements to plan and conduct various skill enhancement trainings and continuous learning opportunities for staff to nourish their skills, knowledge and techniques, ensure proper implementation of patient preparation protocols, setting new guidelines to reduce the rejection and to keep an eye with new trends and advances in medical imaging so, that a reliable image quality is obtained without exposing patients to unnecessary doses of radiation, jeopardize staff protection and burden on the facility and equipments and to cut expenses as well keeping rejection rate as low as possible.

\section{Limitations}

This study has some limitations;

1. The primary one is that it does not includes the number of procedures conducted in the emergency department where high volume of patient coming with trauma and other acute illness undergoing various diagnostic procedures round the clock.

2. Second, it also does not include the number of portable $\mathrm{X}$-ray cases conducted in wards for admitted patients during the study period.

3. There is lack of past rejection data of the facility which could show the actual rejection before the coronavirus disease 2019 (COVID-19) pandemics for comparison. 

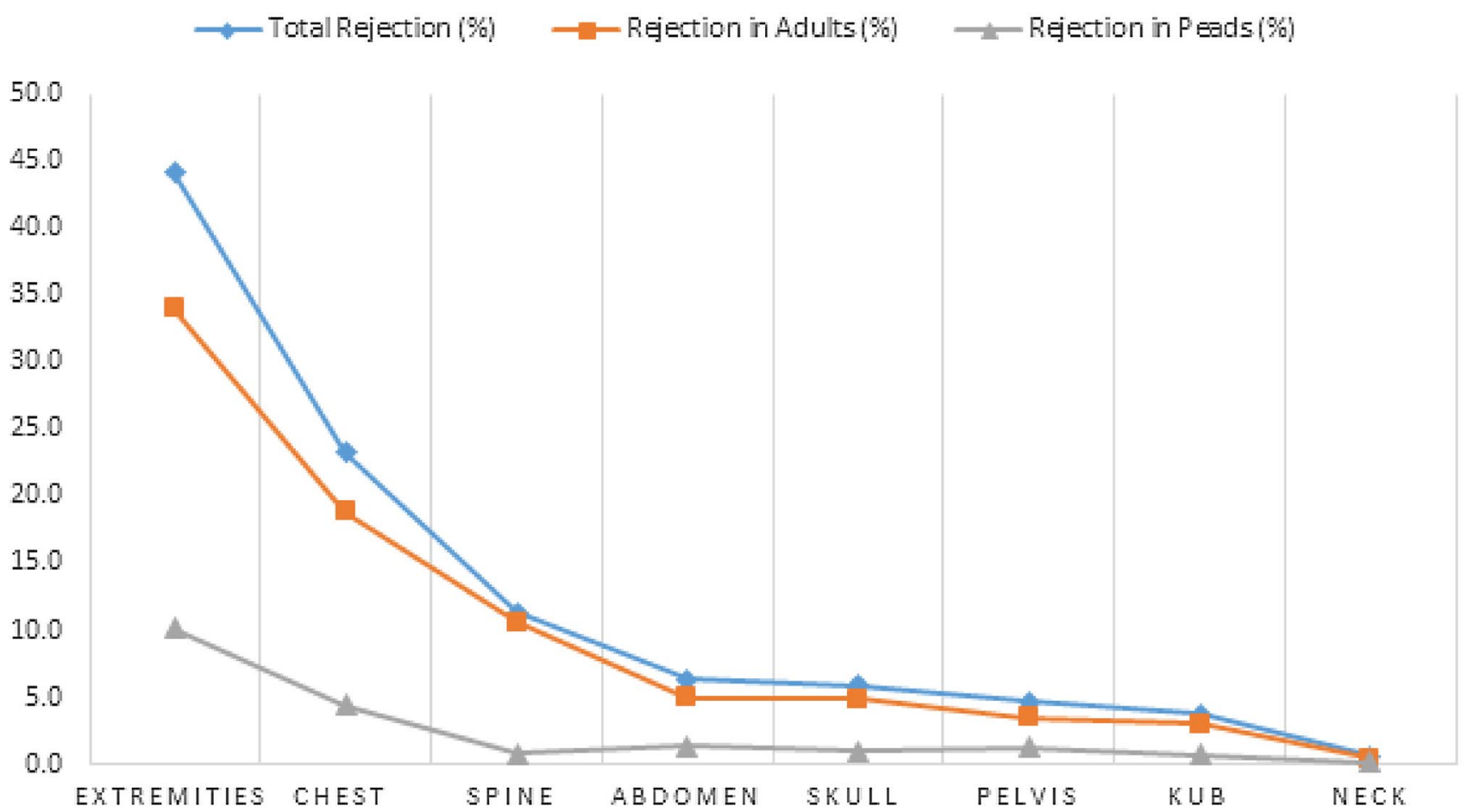

Fig. 5 Comparison of rejection in adults and Peads with respect to body parts

\section{Conclusion}

As this study identified the main causes of radiograph rejection beside the coronavirus disease 2019 (COVID-19) pandemic fear factor which also resulted in high rejection in terms of positioning, patient movement, and anatomical cutoffs in particular. Therefore, reject analysis programs (RAP) should be made integral part of QA program to identify the basic causes of rejection, identify staff needs, and technical gaps, so that comprehensive and specific skill enhancement trainings can be conducted to cut down the number of rejected radiographs and improve good quality diagnostic services along with practical implementation of ALARA principle keeping doses of patient and staff as low as possible.

Acknowledgements The authors wish to acknowledge with thanks, the assistance of the reporting Radiologist on duty, the technical staff, IT and counter staff of the Radiology Department for their cooperation and assistance during data compilation.

Funding The authors disclose no funding source related to this article.

\section{Declarations}

Conflict of interest The authors declare no conflict of interest to this study and declare no relationships with any person/companies whose products or services may be related to the subject matter of the article.
Human/animal rights This article does not contain any human or animal subjects performed by any of the authors.

Informed consent There was no requirement of written informed consent for this study because this was a retrospective study on data with no names or identifiers.

Ethical approval Institutional Ethical Review Board approval was not required because this was a retrospective study on data with no names or identifiers.

\section{References}

1. Jones AK, Heintz P, Geiser W, et al. Ongoing quality control in digital radiography: report of AAPM Imaging Physics Committee Task Group 151. Med Phys. 2015;42:6658-70.

2. Owusu-Banahene J, Darko EO, Hasford F, et al. Film reject analysis and image quality in diagnostic Radiology Department of a Teaching hospital in Ghana. J Radiat Res Appl Sci. 2014;7(4):589-94.

3. Alyousef KA, Alkahtani S, Alessa R, et al. Radiograph reject analysis in a Large Tertiary Care Hospital in Riyadh, Saudi Arabia. Glob J Qual Saf Healthc. 2019;2:30-3.

4. Hofmann B, Rosanowsky TB, Jensen C, et al. Image rejects in general direct digital radiography. Acta Radiol Open. 2015;4:2058460115604339.

5. Khan S, Zahir MZ, Khan J, et al. Frequency of common causes of rejected/repeated chest X-rays in radiology department of a teaching hospital. Gomal J Med Sci. 2016;14:164-6. 
6. Waaler D, Hofmann B. Image rejects/retakes-radiographic challenges. Radiat Prot Dosim. 2010;139:375-9.

7. Shepard SJ, Wang J, Flynn M, et al. An exposure indicator for digital radiography: AAPM task group 116 (executive summary). Med Phys. 2009;36:2898-914.

8. Foos DH, Sehnert WJ, Reiner B, et al. Digital radiography reject analysis: data collection methodology, results, and recommendations from an in-depth investigation at two hospitals. J Digit Imaging. 2009;22:89-98.

9. Zewdu M, Kadir E, Berhane M. Analysis and economic implication of X-ray film reject in Diagnostic Radiology Department of Jimma University Specialized Hospital, Southwest Ethiopia. Ethiop J Health Sci. 2017;27(4):421-6.
10. Andersen ER, Jorde J, Taoussi N, et al. Reject analysis in direct digital radiography. Acta Radiol. 2012;53(2):174-8.

11. Shabestani Monfared A, Abdi R, Saber MA. Repeat analysis program in radiology departments in Mazandaran provinceIran: impact on population radiation dose. Iran J Radiat Res. 2007;5(1):37-40.

12. Lin C-S, Chan P-C, Huan K-H, et al. Guidelines for reducing image retakes of general digital radiography. Adv Mech Eng. 2016;8(4):1-6.

13. Weatherburn GC, Bryan S, West M. A comparison of image reject rates when using film, hard copy computed radiography and soft copy images on picture archiving and communication systems (PACS) workstations. Br J Radiol. 1999;72:653-60. 